\title{
Probing quantum criticality using nonlinear Hall effect in a metallic Dirac system
}

\author{
Habib Rostami and Vladimir Juričić \\ Nordita, KTH Royal Institute of Technology and Stockholm University, Roslagstullsbacken 23, 10691 Stockholm, Sweden
}

(Received 11 October 2019; revised manuscript received 18 December 2019; published 23 January 2020)

\begin{abstract}
Interaction-driven symmetry breaking in a metallic (doped) Dirac system can manifest in the spontaneous gap generation at the nodal point buried below the Fermi level. Across this transition linear conductivity remains finite, making its direct observation difficult in linear transport. We propose the nonlinear Hall effect as a direct probe of this transition when inversion symmetry is broken. Specifically, for a two-dimensional Dirac material with a tilted low-energy dispersion, we predict a transformation of the characteristic interband resonance peak into a non-Lorentzian form in the collisionless regime. Furthermore, we show that inversion-symmetry-breaking quantum phase transition is controlled by an exotic tilt-dependent line of critical points. As this line is approached from the ordered side, the nonlinear Hall conductivity is suppressed owing to the scattering between the strongly coupled incoherent fermionic and bosonic excitations. Our results should motivate further studies of nonlinear responses in strongly interacting Dirac materials.
\end{abstract}

DOI: 10.1103/PhysRevResearch.2.013069

\section{INTRODUCTION}

Nonlinear response functions are extremely sensitive to the structural symmetry of crystalline systems. In particular, the second-order spectroscopy, such as second harmonic generation (SHG), probing the second-order conductivity, is a powerful technique to characterize the crystalline orientation of a sample [1]. The SHG is forbidden in the presence of spatial inversion symmetry and can therefore play the role of an order parameter distinguishing the phases across the transition at which the spatial inversion symmetry is broken. Furthermore, there has recently been growing interest in the nonlinear (second-order) Hall effect [2-5] which, unlike the usual one, occurs in the presence of time-reversal symmetry in noncentrosymmetric (semi)metals featuring tilted Dirac fermions (TDFs) at low energies, such as single- and fewlayer $\mathrm{WTe}_{2}$ [6-8]. The nonlinear Hall effect amounts to the generation of a transverse current as a second-order response to a linearly polarized external electric field and, as has been recently shown, it is controlled by the Fermi surface average of the Berry curvature derivative, the so-called Berry curvature dipole [5,9-12]. Other phenomena, e.g., injection and anomalous photocurrent in Weyl semimetals, are also interesting nonlinear phenomena related to the Berry curvature dipole [9,13-17].

In this work we show that the nonlinear Hall effect can be used as a powerful tool to probe the electron-interactiondriven inversion-symmetry breaking in a metallic phase that emerges from a generic nodal band structure. In this case, the chemical potential is outside the gap region (see Fig. 1) and

Published by the American Physical Society under the terms of the Creative Commons Attribution 4.0 International license. Further distribution of this work must maintain attribution to the author(s) and the published article's title, journal citation, and DOI. the usual linear conductivity is finite in both symmetric and symmetry-broken phases. In contrast, the nonlinear conductivity is finite only when the gap is opened, i.e., the inversion symmetry is broken, and therefore may be used to directly detect the phase transition.

The inversion-symmetry breaking at a finite chemical potential $\mu>0$ strongly relies on the presence of a Dirac point buried below the Fermi level. Namely, at a finite chemical potential (but low enough so that the Dirac approximation is still valid) and at a strong short-range (Hubbard-like) electron interaction, the band-gap opening may occur at the Dirac point because the system would optimally deplete the free energy as it would for $\mu=0$. The latter is expected since quite generically there is no phase space for an interaction-driven Mott insulating instability to take place at a finite but low enough $\mu$ [18]. Irrespective of whether the system is electron doped

\section{Inversion symmetric metal Inversion breaking metal}

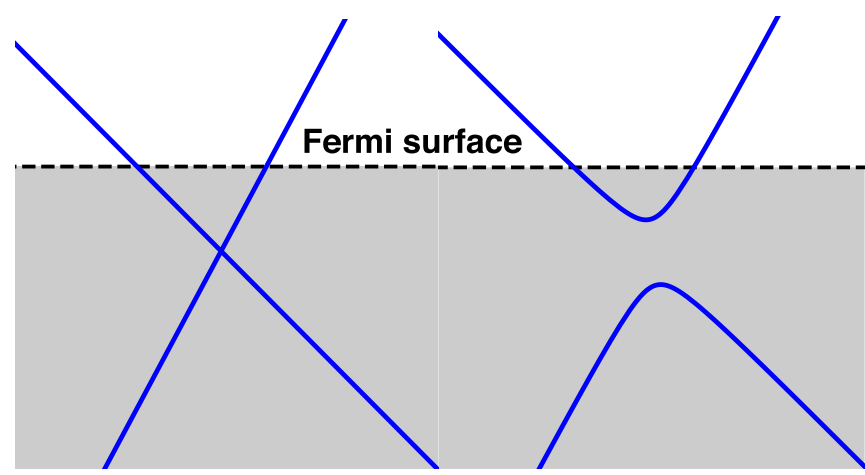

FIG. 1. Schematic plot for an inversion symmetric and inversion breaking semimetal described by tilted Dirac fermions at low energies. Although the gap opening occurs away from the Fermi surface, it can have a strong impact on the nonlinear response of the system in the corresponding metallic phase $(\mu>0)$. 
$(\mu>0)$ or hole doped $(\mu<0)$, this scenario is expected to remain operative up to a critical chemical potential, at which a superconducting instability takes over (see Ref. [19] for a discussion of the stability of a doped interacting Dirac liquid without the tilt).

We therefore consider strongly interacting twodimensional TDFs at the neutrality point $(\mu=0)$ and at zero temperature $(T=0)$ in the vicinity of a quantum phase transition (QPT) into an inversion-symmetry broken phase within the framework of the Gross-Neveu-Yukawa (GNY) quantum-critical theory. It is well established that the GNY field theory captures the behavior of strongly interacting gapless Dirac fermions in the vicinity of a QPT to an ordered gapped phase [20-27]. Furthermore, the field-theoretic predictions have been corroborated by the results from the numerical (mostly quantum Monte Carlo) simulations [28-31]. By employing a renormalization-group (RG) analysis to the leading order in the $\epsilon(=3-D)$ expansion close to the upper critical $D=3$ spatial dimensions, we show that in the presence of the tilt a QPT into an inversionsymmetry-broken phase is controlled by an unusual tilt-dependent line of the quantum-critical points (QCPs) which may be stable nonperturbatively, due to the pseudorelativistic invariance of the boson-fermion Yukawa interaction and the specific form of the tilt term.

We find that the dynamical nonlinear Hall conductivity (NLHC) is suppressed in the ordered (symmetry-broken) phase close to this line of QCPs as compared to the noninteracting massive TDFs and therefore can be used to probe such a symmetry breaking in a TDF metal. This effect can be traced back to the scattering of the strongly interacting soup of incoherent fermionic and bosonic excitations close to this line of QCPs. Furthermore, this suppression increases with the tilt parameter, consistent with the expectation based on the scaling of the density of states (DOS).

The paper is organized as follows. In Sec. II we present a general scaling analysis of nonlinear conductivity in Dirac systems. In Sec. III we analyze the main features of the second-order conductivity of two-dimensional TDFs in the collisionless regime and zero temperature. In Sec. IV we introduce the GNY quantum-critical theory for TDFs, while in Sec. IV A we perform the leading-order RG analysis of the theory and in Sec. IV B compute the interaction correction to the nonlinear conductivity at the line of strongly coupled QCPs. Material aspects of our proposal are discussed in Sec. V. We summarize our work in Sec. VI.

\section{NONLINEAR CONDUCTIVITY: SCALING ANALYSIS}

Dimensional analysis of the nonlinear conductivity in a $D$-dimensional Dirac system with linear dispersion (the dynamical exponent $z=1$ ) yields $\operatorname{dim}\left[\sigma^{(n)}\right]=D-2 n$ in units of momentum (inverse length), as shown in Appendix A. We only address the collisionless regime where frequency $\omega \gg T$, which is governed by the particle-hole excitations created by the external electric field, since in this regime the conductivity displays universal features dictated exclusively by the dimensionality, the dispersion of the low-energy quasiparticle excitations, and the electron-electron interactions [32]. For the TDFs at finite temperature and frequency, the scaling form of the nonlinear optical conductivity reads (see Appendix A)

$$
\sigma^{(n)}(n \omega) \sim \frac{1}{\omega^{2 n-D}} f^{(n)}\left(\frac{\omega}{T}, \frac{\mu}{T}, \frac{m_{f}}{T}, \alpha,\{g\}\right),
$$

where $f^{(n)}(\{X\})$ is a universal scaling function of the dimensionless parameters $\{X\}, \mu$ and $m_{f}$ stand for the chemical potential and fermion mass, respectively, and $\alpha$ and $\{g\}$ represent the tilt parameter and dimensionless couplings. We here only focus on the high harmonic generation case for which all the frequencies are equal, and for the notational clarity we use $\sigma^{(n)}\left(\omega_{1}=\omega, \omega_{2}=\omega, \ldots, \omega_{n}=\omega\right) \equiv \sigma^{(n)}(n \omega)$.

In the proximity of the line of strongly coupled QCPs, given by Eq. (12), which, as we show, governs the behavior of the TDFs at the QPT, the nonlinear conductivity picks up a correction given by

$$
\sigma_{*}^{(n)}(n \omega)=Z_{\Psi}^{n+1} \sigma^{(n)}(n \omega) .
$$

Here $Z_{\Psi}$ is the renormalization factor for the fermionic field at this line of QCPs, which is directly related to the corresponding anomalous dimension, and $\sigma^{(n)}(n \omega)$ is the nonlinear conductivity for the noninteracting massive TDFs. Vertex corrections are absent due to the gauge invariance. The case of $n=1$ and $\alpha=0$ corresponds to the linear conductivity of the untilted Dirac fermions [33]. We show that for the $T=0$ SHG $(n=2)$ the correction explicitly reads

$$
\sigma_{*}^{(2)}(2 \omega)=\left\{1-\frac{3}{N_{f}\left(4-\alpha^{2}\right)}\right\} \sigma^{(2)}(2 \omega)
$$

to the leading order in the $\epsilon$ and $1 / N_{f}$ expansions, with $\sigma^{(2)}(2 \omega)$ as the $T=0$ second-order conductivity of the noninteracting system, given by Eq. (6). We note that the conductivity is suppressed as compared to the noninteracting system due to the strong interactions of the fermionic and the order-parameter (bosonic) fluctuations close to the line of QCPs. The suppression is a monotonically increasing function of the tilt parameter which is consistent with the increase of the DOS at any finite energy and at a finite tilt with respect to the untilted case. Furthermore, we obtain universal interband features in the NLHC: non-Lorentzian resonance peaks in the collisionless regime stemming from the anisotropic Fermi surface at the finite tilt, with the position and the linewidth strongly dependent on the tilt parameter. We note that the strong tilt dependence of the linear optical properties of TDFs was previously discussed [34,35].

\section{SECOND-ORDER CONDUCTIVITY OF NONINTERACTING TILTED DIRAC FERMIONS}

We consider an external homogeneous vector potential $\mathbf{A}(t)$, with the corresponding electric field $\mathbf{E}(t)=-\partial_{t} \mathbf{A}(t)$, as the driving field. The local second-order conductivity is obtained by utilizing the Kubo formula

$$
\sigma_{a b c}^{(2)}\left(\omega_{1}, \omega_{2}\right)=-\left.\frac{\chi_{a b c}^{(2)}\left(i \Omega_{1}, i \Omega_{2}\right)}{\omega_{1} \omega_{2}}\right|_{i \Omega_{m} \rightarrow \omega_{m}+i \delta} .
$$

Note that $\delta \rightarrow 0^{+}, m=1,2$, and the second-order susceptibility given in terms of a three-point imaginary-time correlation function $\chi_{a b c}^{(2)}\left(\tau_{1}, \tau_{2}\right)=\left\langle\mathcal{T} \hat{j}_{c}\left(-\tau_{2}\right) \hat{j}_{b}\left(-\tau_{1}\right) \hat{j}_{a}(0)\right\rangle / 2$, where $\mathcal{T}$ 
stands for the time-ordering operation and $\hat{j}_{a}=-e \sum_{\mathbf{k}}$ $\hat{\psi}_{\mathbf{k}}^{\dagger} \partial_{k_{a}} \hat{\mathcal{H}}_{\mathbf{k}} \hat{\psi}_{\mathbf{k}}$. The paramagnetic current in the interaction picture $\hat{j}_{a}(\tau)=e^{-\tau \hat{\mathcal{K}}} \hat{j}_{a} e^{\tau \hat{\mathcal{K}}}$, with $\hat{\mathcal{K}}=\sum_{\mathbf{k}} \hat{\psi}_{\mathbf{k}}^{\dagger}\left(\hat{\mathcal{H}}_{\mathbf{k}}-\mu \hat{I}\right) \hat{\psi}_{\mathbf{k}}$, corresponds to the single-particle Hamiltonian $(\hbar=1)$

$$
\hat{\mathcal{H}}(\mathbf{k})=\alpha \zeta k_{x} \hat{I}+v\left(\zeta k_{x} \hat{\sigma}_{x}+k_{y} \hat{\sigma}_{y}\right)+m_{f} \hat{\sigma}_{z},
$$

where $\zeta= \pm$ stands for two fermion flavors, $m_{f}$ represents the fermion mass due to the inversion-symmetry breaking, $\hat{\sigma}$ are the Pauli matrices, $\hat{I}$ is the $2 \times 2$ unity matrix, and the Fermi velocity $v=1$ hereafter.

We first calculate the NLHC $\sigma_{y x x}^{(2)}$ in response to a linearly polarized electric field along the $x$ direction. In principle, there are DC and AC contributions which correspond to rectification and second harmonic effects, respectively. We here focus on the latter, since we consider the collisionless regime. The zero-temperature NLHC of an electron-doped system is given in terms of the Berry curvature through its derivative (see Appendix B)

$$
\sigma_{y x x}^{(2)}(2 \omega)=\frac{i e^{3}}{\omega} \sum_{\mathbf{k}, \zeta} n_{\mathrm{F}}\left(\varepsilon_{\mathbf{k}}^{c}\right) \frac{\partial \Omega_{y x}(k)}{\partial k_{x}} C\left(\frac{\omega}{2 \varepsilon_{k}}\right) .
$$

Here $n_{\mathrm{F}}(\varepsilon)=\Theta(\mu-\varepsilon)$ is the Fermi-Dirac distribution function at $T=0, \varepsilon_{\mathbf{k}}^{c}=\zeta \alpha k_{x}+\varepsilon_{k}$, with $\varepsilon_{k}=\sqrt{k^{2}+m_{f}^{2}}$, is the conduction band dispersion, $\Omega_{y x}(k)=\zeta m_{f} / 2 \varepsilon_{k}^{3}$ stands for the Berry curvature [36], and $C(x)=1 /\left[\left(1-4 x^{2}\right)\left(1-x^{2}\right)\right]$. We see that even though the system is metallic, there is a strong interband correction to the NLHC, characteristic of the collisionless regime.

The NLHC in the noninteracting system, after a momentum integration in Eq. (6), reads explicitly

$$
\sigma_{y x x}^{(2)}(2 \omega)=\frac{i e^{3}}{\omega^{2}} f\left(\frac{\omega}{\mu}, \frac{m_{f}}{\mu}, \alpha\right),
$$

where the universal function is to leading order in $\alpha$ given by (see Appendix C)

$$
f(x, y, \alpha) \approx \frac{12 \alpha}{\pi^{2}} \frac{x y\left(1-y^{2}\right) \Theta(1-y)}{\left(x^{2}-1\right)\left(x^{2}-4\right)} .
$$

In the case of $\alpha \ll 1$, the interband resonances occur at $x=\omega / \mu \approx 1$ and $x=\omega / \mu \approx 2$. The exact dependence of $f(x, y, \alpha)$ on its arguments for $0 \leqslant \alpha \leqslant 1$ is given by a quite lengthy analytical expression explicitly displayed in Appendix C, plotted in Fig. 2.

The corresponding result for the intraband regime $\omega<\mu$ is depicted in Fig. 2(a) and the $f$ function is real valued, similar as in Ref. [5]. Its form in the interband regime $\omega>\mu$ is displayed in Figs. 2(b)-2(d), where we can see that both the position and the shape of interband resonances strongly depend on the value of $\alpha$. This effect can be explained by considering the anisotropic Fermi surface, which leads to a momentum-dependent optical gap $\Delta(\mathbf{k})$ for the finite- $\alpha$ case. The single-photon and multiphoton resonances occur when $n \omega$, with $n=1,2, \ldots$, is equal to the optical gap at each momentum. Explicitly, in the presence of the tilt, the optical gap $\Delta(\mathbf{k})=2 \sqrt{m_{f}^{2}+k^{2}}$, where $\mathbf{k}=\left(k_{x}, k_{y}\right)$ runs over the Fermi surface $\left(k_{x}+\zeta k_{0}\right)^{2}+k_{y}^{2} /\left(1-\alpha^{2}\right)=R^{2}$ in which $k_{0}=$ $\alpha \mu /\left(1-\alpha^{2}\right)$ and $R=\sqrt{\mu^{2}-\left(1-\alpha^{2}\right) m_{f}^{2}} / \sqrt{1-\alpha^{2}}$. Such an optical gap at finite $\alpha$ leads to a splitting of the interband
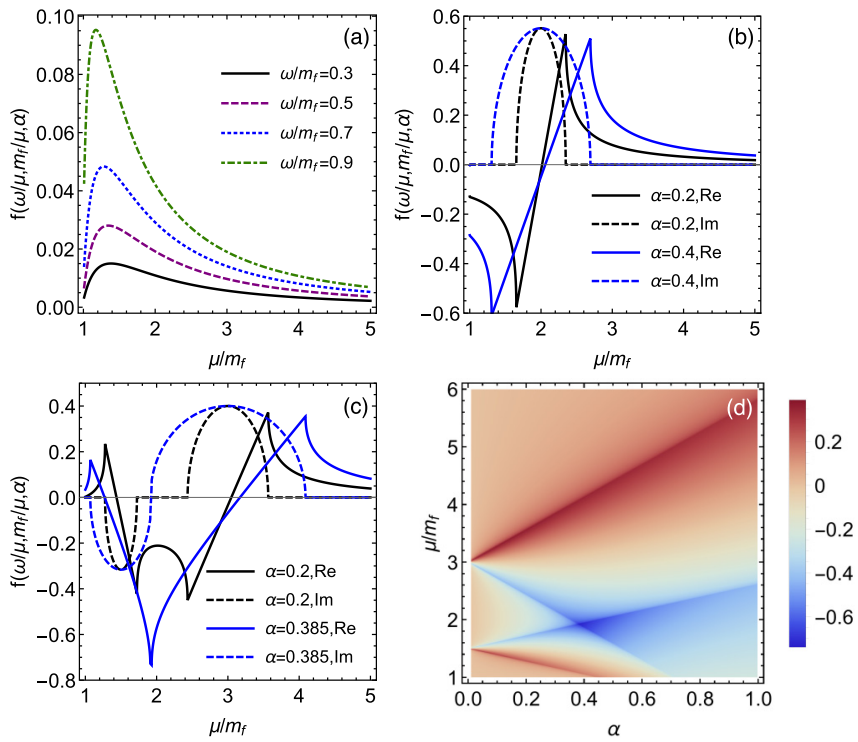

FIG. 2. Dependence of the universal function $f$, defined in Eq. (7), with respect to $\mu / m_{f}$ and $\alpha$ for several values of $\omega / m_{f}$, as calculated in Appendix C. (a) Intraband regime for which $\omega<\mu$ and we set $\alpha=0.2$. The function $f$ is purely real in this regime. (b) Interband regime with $\omega=2 m_{f}$. We can see that the position of peaks and dips strongly depends on $\alpha$ and an imaginary part in the resonance region appears. (c) Interband regime with $\omega=3 m_{f}$ for which there are two resonances. At a specific value of $\alpha \approx 0.385$ two dips merge. (d) Color plot for the real part of the function $f$ at $\omega=3 m_{f}$ in which the shift in the position of peaks and dips can be traced.

resonance peaks where the dip and the peak of the real part of the $f$ function shift in opposite directions. Simultaneously, a broad non-Lorentzian resonance feature emerges in the imaginary part of the $f$ function (see Appendix D for a detailed discussion). For $\alpha \ll 1$, the Fermi surface is almost a circle with radius $k_{\mathrm{F}}$, which is the Fermi wave vector in the absence of the tilt. Therefore, the optical gap is nearly independent of the momentum $\Delta(\mathbf{k})=2 \sqrt{m_{f}^{2}+k_{\mathrm{F}}^{2}}=2 \mu$. Accordingly, the interband resonances are quite sharp for the case of $\alpha \ll 1$. Another nontrivial feature of the universal $f$ function is the cusplike resonances [see Figs. 2(b) and 2(c)] in its real part stemming from its logarithmic form, as explicitly given in Appendix C [see Eq. (C21)]. The corresponding one- and twophoton resonances shift in the opposite directions in frequency by increasing the value of the tilt parameter $\alpha$. At a critical value of $\alpha$, the two resonances morph into a single one and then pass each other upon a further enhancement of $\alpha$, as can be seen in Fig. 2(d).

\section{GROSS-NEVEU-YUKAWA QUANTUM-CRITICAL THEORY FOR TILTED DIRAC FERMIONS}

We now consider the effect of a strong short-range (Hubbard-like) electron interaction on the nonlinear optical conductivity within the framework of the GNY quantumcritical theory for the TDFs. The space-imaginary-time action of the theory is $S=S_{f}+S_{Y}+S_{b}$ and the noninteracting 
fermionic part reads

$$
S_{f}=\int d \tau d^{D} \mathbf{r} \psi^{\dagger}\left[\partial_{\tau}+\hat{\mathcal{H}}\left(\mathbf{k} \rightarrow-i \nabla_{\mathbf{r}}\right)\right] \psi,
$$

where the Dirac fermion field $\psi \equiv \psi(\tau, \mathbf{r})$ and $\hat{\mathcal{H}}(\mathbf{k})$ is the Hamiltonian for the noninteracting TDFs in Eq. (5) with $m_{f}=0$. The summation over the valley degree of freedom is assumed and we consider $2 N_{f}$ copies of two-component Dirac spinors hereafter. The short-range interaction is encoded through the Yukawa coupling between the Dirac fermion quasiparticles and the fluctuations of the underlying ordered state assumed to break Ising $\left(Z_{2}\right)$ symmetry, such as a sublattice symmetry-breaking charge density wave in graphene,

$$
S_{Y}=g \int d \tau d^{D} \mathbf{r} \phi \psi^{\dagger} \hat{\sigma}_{z} \psi
$$

Here the bosonic field $\phi \equiv \phi(\tau, \mathbf{r})$ with the dynamics given by the action

$$
S_{b}=\int d \tau d^{D} \mathbf{r}\left\{\phi\left(-\partial_{\tau}^{2}-\nabla^{2}+m_{b}^{2}\right) \phi+\frac{\lambda}{4 !} \phi^{4}\right\},
$$

where $m_{b}^{2}$ is the tuning parameter for the transition and $m_{b}^{2}>0$ $(<0)$ in the symmetric (symmetry broken) phase.

Engineering scaling dimensions of the Yukawa and $\phi^{4}$ couplings are $\operatorname{dim}\left[g^{2}\right]=\operatorname{dim}[\lambda]=3-D$, while for the tilt parameter $\operatorname{dim}[\alpha]=0$, implying that $D=3$ is the upper critical dimension in the theory. We therefore use the deviation from the upper critical dimension as an expansion parameter $\epsilon=3-D$ to access the quantum-critical behavior in $D=2$. We set the bosonic and fermionic velocities to be equal to unity in the critical region [37].

\section{A. Renormalization-group analysis: Line of quantum-critical points}

To obtain the RG flow of the couplings, we integrate out the modes with the Matsubara frequency $-\infty<\omega<\infty$ and then use the dimensional regularization in $D=3-\epsilon$ spatial dimensions within the minimal subtraction scheme. The RG $\beta$ functions to the leading order in the $\epsilon$ expansion, in the critical hyperplane $\left(m_{b}^{2}=0, m_{f}=0\right)$, read (see Appendix E)

$$
\begin{aligned}
\beta_{g^{2}} & =\epsilon g^{2}-g^{4} K\left(\alpha, N_{f}\right), \\
\beta_{\lambda} & =\epsilon \lambda-\frac{3}{2} \lambda^{2}-4 N_{f}\left(1+2 \alpha^{2}\right) \lambda g^{2}+24 g^{4} N_{f}, \\
\beta_{\alpha} & =0,
\end{aligned}
$$

with $K\left(\alpha, N_{f}\right)$ calculated in Appendix $\mathrm{E}$ and displayed in Fig. 3, $\beta_{Y} \equiv-d Y / d \ln (\kappa), \kappa$ the RG (momentum) scale, and the couplings rescaled as $X / 8 \pi^{2} \rightarrow X$, with $X \in\left\{g^{2}, \lambda\right\}$. In the limit $\alpha \rightarrow 0$ it can be readily seen that these $\beta$ functions reduce to the well known ones for the Ising GNY theory [22,38]. Crucially, for any $|\alpha|<1$, due to the marginality of the tilt parameter, the above flow equations yield a line of QCPs, given by

$$
\left(g_{\star}^{2}, \lambda_{\star}\right)=\epsilon\left(\frac{1}{K\left(\alpha, N_{f}\right)}, h\left(\alpha, N_{f}\right)\right),
$$

with $h\left(\alpha, N_{f}\right)$ a complicated function of its arguments (see Appendix E). Note that $K\left(\alpha, N_{f}\right)$ is a strictly positive and

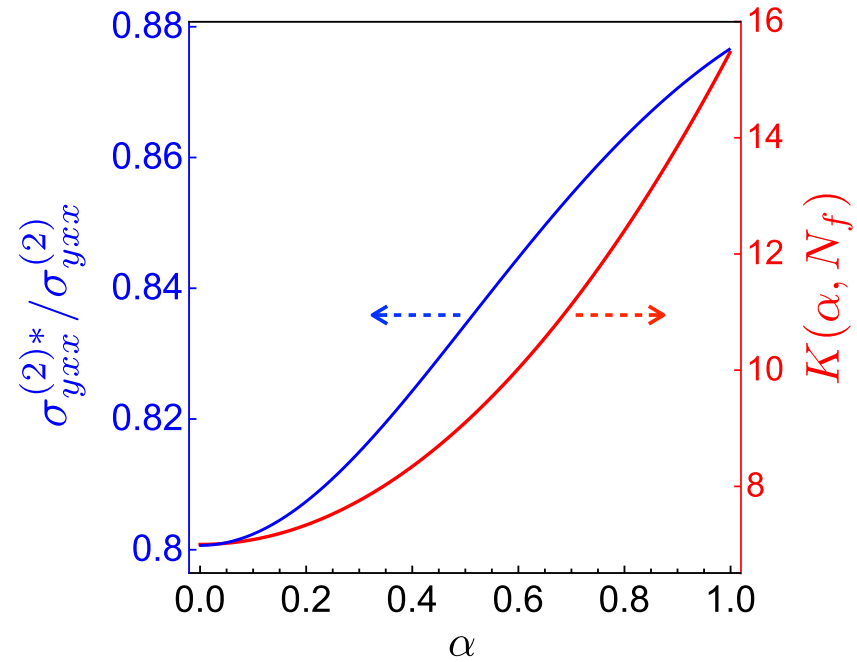

FIG. 3. Universal function $K\left(\alpha, N_{f}\right)$ and corrected NLHC relative to its bare value $\sigma_{y x x}^{(2) *} / \sigma_{y x x}^{(2)}$, shown as a function of the tilt parameter $\alpha$ in the spinless case $N_{f}=2$.

monotonically increasing function for $0 \leqslant \alpha<1$, and therefore the value of the Yukawa coupling at the QCPs is smaller in comparison with untilted Dirac fermions; the same holds for the $\phi^{4}$ coupling $\lambda$. This can be understood from the fact that the density of states $\rho(E, \alpha) \sim|E| / \sqrt{1-\alpha^{2}}$, and therefore at any finite energy the DOS for TDFs increases as compared to untilted Dirac fermions until the system is overtilted at $|\alpha|=1$. It is thus expected that the location of the critical point is pushed to a weaker coupling as the tilt increases and this feature is indeed captured in the RG analysis. However, the DOS is still vanishing at zero energy for a finite tilt, implying that the critical points remain at a finite coupling, again consistent with the RG analysis. At this line of QCPs, both fermionic and bosonic excitations cease to exist as sharp quasiparticles because of their nontrivial anomalous dimension given by $\eta_{\Psi}=g_{\star}^{2} G(\alpha) / 2$ and $\eta_{\Phi}=$ $2 N_{f}\left(1+2 \alpha^{2}\right) g_{\star}^{2}$, respectively, with $G(\alpha)=4 /\left(4-\alpha^{2}\right)$, obtained from $\eta_{i}=-\left(d Z_{i} / d g^{2}\right) \beta_{g^{2}}$ calculated at $X=X_{\star}$, with $Z_{i}\left(g^{2}\right), i=\Psi, \Phi$, as the leading-order field renormalizations (see Appendix E). Therefore, a family of non-Fermi liquids emerges from the QCP at a finite temperature for any $|\alpha|<1$.

We would like to emphasize that the marginality of the tilt parameter $\alpha$ as given by Eq. (14) may be nonperturbative in nature, implying that the line of QCPs we found to the leading order in the $\epsilon$ expansion may be stable beyond this order. The reason for this lies in the pseudorelativistic invariance of the Yukawa interaction [see Eq. (E3) and the discussion thereof], given in Eq. (10), and the specific form of the tilt term. Namely, the tilt term manifestly breaks this symmetry and commutes with the rest of the free fermion action, as well as with the matrix entering the Yukawa term. Therefore, the Yukawa term is expected not to renormalize it, implying that the tilt parameter remains marginal. On the other hand, it was shown that manifestly Lorentz symmetry-breaking long-range Coulomb interaction renders the tilt parameter irrelevant, consistent with the above argument [39]. 


\section{B. Interaction correction to the nonlinear conductivity at the line of quantum-critical points}

The leading-order correction to the conductivity in the vicinity of the QCP is determined by the fermionic field renormalization $Z_{\Psi}$, as given by Eq. (2), which is ultimately related to the fermionic self-energy at vanishing external momentum $\Sigma_{f}(i \Omega)$, explicitly evaluated in Appendix E, yielding

$$
Z_{\Psi}=1-g_{\star}^{2} \frac{1}{2 \epsilon} G(\alpha) .
$$

Therefore, the nonlinear conductivity is modified according to Eq. (2) due to a strong interaction between incoherent fermionic and bosonic excitations close to the QCPs. Explicitly, the form of the correction to the NLHC reads

$$
\sigma_{y x x}^{(2) *}(2 \omega)=\left(1-\frac{G(\alpha)}{2 K\left(\alpha, N_{f}\right)}\right)^{3} \sigma_{y x x}^{(2)}(2 \omega),
$$

which is displayed in Fig. 3, and in the large- $N_{f}$ limit yields the result shown in Eq. (1).

\section{MATERIAL REALIZATIONS}

The case of $\mathrm{WTe}_{2}$ is particularly interesting because of a very recent experimental observation of nonlinear Hall effect [6-8]. Actually, $\mathrm{WTe}_{2}$ without the spin-orbit coupling can be described in terms of tilted massless Dirac fermions $[7,40]$, while the spin-orbit coupling opens up a direct gap located at $Q$ and $Q^{\prime}$ valley points in the Brillouin zone $[7,41]$ with the Berry curvature hot spots localized around these points [5,9-12]. Considering the tilt parameter $\alpha \approx 0.1$, the Fermi velocity $v \approx 0.5 \times 10^{6} \mathrm{~m} / \mathrm{s}$, the Dirac mass $m_{f} \approx$ $75 \mathrm{meV}$, the chemical potential $\mu \approx 1.3 m_{f} \sim 100 \mathrm{meV}$, and at $\hbar \omega \approx 120 \mathrm{meV}$ [6-8], we estimate the noninteracting NLHC to be $\left|\sigma^{(2)}(2 \omega)\right|=\sigma_{0} / E_{0}$, where $\sigma_{0}=e^{2} / \hbar$ and $E_{0}=$ $(\hbar \omega)^{2} / e \hbar v|f| \approx 0.31 \mathrm{~V} / \mathrm{nm}$, with $|f| \approx 0.14$. On the other hand, if this value of the mass gap is induced by the strong short-range interaction close to the QCP, the NLHC should decrease as compared to this result for the noninteracting gapped (massive) TDFs, according to Eq. (17).

There are several other candidates for the realization of massless TDFs in two dimensions such as 8-Pmmn borophone [42,43] with an electrically tunable tilt strength [44], topological crystalline insulators such as SnTe [45], and an organic compound such as $\alpha$-(BEDT-TTF $)_{2} \mathrm{I}_{3}$ under pressure [46-48]. Strong short-range electron interactions, such as the on-site Hubbard interaction, may catalyze a mass gap therein, and the predicted behavior of the nonlinear Hall conductivity can be used to probe this phase transition. Furthermore, an analog of twisted bilayer graphene featuring tilted and slow Dirac fermions at low energies may be an ideal candidate to realize the scenario we proposed in our work. Finally, in three spatial dimensions, being the upper critical dimension for the GNY theory, only a correction to the conductivity stemming from the long-range Coulomb interaction should remain $[33,49]$. This correction is expected to be $\alpha$ independent due to the irrelevance of the tilt parameter in this case [39].

\section{SUMMARY AND OUTLOOK}

To summarize, we have proposed NLHC as an efficient tool to probe interaction tuned inversion-symmetry breaking in the materials featuring Dirac fermions with the tilted dispersion. We showed that the quantum-critical behavior at a strong short-range interaction is governed by a line of QCPs from which a family of non-Fermi liquids emerges at a finite temperature. We found that NLHC decreases as the system approaches this line of QCPs from the ordered (symmetrybroken) phase. Our results should motivate further studies of nonlinear response functions in strongly correlated Dirac materials, such as the organic compound $\alpha$-(BEDT-TTF $)_{2} \mathrm{I}_{3}$ [48]. Finally, the family of non-Fermi liquids we uncovered here should be further characterized in terms of optical and thermodynamic responses.

\section{ACKNOWLEDGMENT}

H.R. acknowledges support from the Swedish Research Council (Grant No. VR 2018-04252).

\section{APPENDIX A: DIMENSIONAL ANALYSIS}

The light-matter interaction is defined by the interaction term in the action

$$
S_{\text {int }} \sim \int d^{D} x d t \mathbf{J} \cdot \mathbf{A},
$$

where $D$ is the spatial dimension, $\mathbf{A}$ is the vector potential, and the corresponding electric field reads $\mathbf{E}=-\partial_{t} \mathbf{A}$. The current is given as the sum of linear and all nonlinear contributions

$$
\mathbf{J} \sim \sum_{n \geqslant 1} \boldsymbol{\sigma}^{(n)} \underbrace{\mathbf{E E} \ldots \mathbf{E}}_{n \text { times }} .
$$

Note that the dimension of the parameters in the units of momentum (inverse length) is

$$
\begin{aligned}
& \operatorname{dim}\left[S_{\text {int }}\right]=0, \quad \operatorname{dim}\left[x_{\mu}\right]=-1, \quad \operatorname{dim}\left[A_{\mu}\right]=1, \\
& \operatorname{dim}[t]=-\operatorname{dim}[\omega]=-z,
\end{aligned}
$$

where $z$ stands for the dynamical exponent which implies an energy dispersion as $\varepsilon_{k} \sim k^{z}$. Therefore, we have

$$
\begin{aligned}
& \operatorname{dim}\left[E_{\mu}\right]=\operatorname{dim}\left[A_{\mu}\right]-\operatorname{dim}[t]=1+z, \\
& \operatorname{dim}\left[J_{\mu}\right]=D+z-1 .
\end{aligned}
$$

Eventually, we obtain

$$
\operatorname{dim}\left[\sigma^{(n)}\right]=D-(n+1)-(n-1) z .
$$

For the Dirac model we have $z=1$, which implies $\operatorname{dim}\left[\sigma^{(n)}\right]=D-2 n$, and thus the scaling form of the $n$ thorder conductivity reads

$$
\sigma^{(n)} \sim \frac{1}{\omega^{2 n-d}} f^{(n)}\left(\frac{\omega}{T}, \frac{\mu}{T}, \alpha,\{g\}\right) .
$$

Note that the linear conductivity of (undoped or intrinsic) Dirac systems in $D=2$ is dimensionless, while the secondorder one scales as $1 / \omega^{2}$. This implies that at low-frequency spectroscopy like $\mathrm{GHz}$ or even $\mathrm{THz}$ the nonlinear response can be considerably stronger than its linear counterpart and may be a very good instrument to probe those phenomena which are hidden within the linear response framework. 


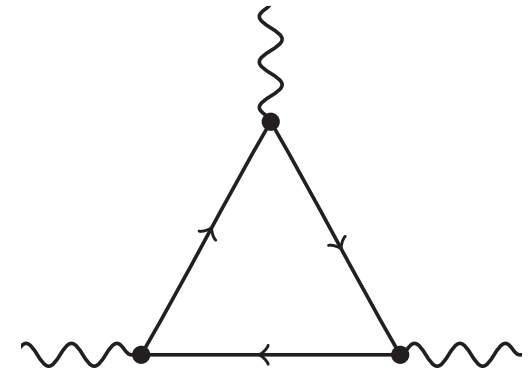

FIG. 4. Feynman diagram for the second-order susceptibility which is a three-point correction function of paramagnetic current operator components. The solid line represents the fermionic propagator while the wavy line stands for the external photons.

\section{APPENDIX B: NONLINEAR HALL CONDUCTIVITY EVALUATED USING THE KUBO FORMULA}

For the Dirac system with the Hamiltonian linearly dependent on the momentum, such as the one we consider here for TDFs given in Eq. (5), there is only one Feynman diagram for the second-order susceptibility $\chi_{\alpha \beta \gamma}^{(2)}$, which is shown in Fig. 4 and is defined as

$$
J_{\alpha}^{(2)}=\sum_{\beta \gamma} \chi_{\alpha \beta \gamma}^{(2)} A_{\beta} A_{\gamma} .
$$

The susceptibility corresponding to this bubble diagram reads [50]

$$
\begin{aligned}
& \chi_{\alpha \beta \gamma}^{(2)}\left(m_{1}, m_{2}\right)=\sum_{\mathcal{P}}^{\prime} \sum_{\mathbf{k}} \frac{1}{\beta} \sum_{n} \operatorname{tr}\left[\hat{\Gamma}_{\alpha} \hat{G}_{f}(\mathbf{k}, n) \hat{\Gamma}_{\beta}\right. \\
& \left.\times \hat{G}_{f}\left(\mathbf{k}, n+m_{1}\right) \hat{\Gamma}_{\gamma} \hat{G}_{f}\left(\mathbf{k}, n+m_{1}+m_{2}\right)\right],
\end{aligned}
$$

where $\sum_{\mathcal{P}}^{\prime}$ stands for the intrinsic permutation symmetry [51], e.g., $\left(\beta, m_{1}\right) \leftrightarrow\left(\gamma, m_{2}\right)$, and the fermionic Green's function is given by

$$
\hat{G}_{f}(\mathbf{k}, n)=[n \hat{I}-\hat{\mathcal{H}}(\mathbf{k})]^{-1} .
$$

Note that $\hat{I}$ is the identity matrix and $n\left(m_{i}\right)$ stands for the shorthand notation of the dummy (external) Matsubara frequency $i \omega_{n}=(2 n+1) \pi / \beta \quad\left(i \omega_{m_{i}}=2 m_{i} \pi / \beta\right)$ and $\beta=$ $1 / k_{\mathrm{B}} T$, where $k_{\mathrm{B}}$ is the Boltzmann constant and $T$ is the temperature. The current vertex functions for the TDF Hamiltonian given in Eq. (5) read

$$
\hat{\Gamma}_{x}=-e \partial_{k_{x}} \hat{\mathcal{H}}=-e \zeta \hat{\sigma}_{x}-e \alpha \zeta \hat{I}, \quad \hat{\Gamma}_{y}=-e \partial_{k_{y}} \hat{\mathcal{H}}=-e \hat{\sigma}_{y} .
$$

It is convenient to proceed with the calculation in the band basis, which is denoted by $|\mathbf{k}, \lambda\rangle$ with $\lambda \equiv \zeta, \ell$, where $\ell=$ $c, v$ represent the conduction and valence bands. We note that

$$
\begin{aligned}
\left\langle\mathbf{k}, \lambda_{1}|\hat{G}(\mathbf{k}, n)| \mathbf{k}, \lambda_{2}\right\rangle & =\frac{\delta_{\lambda_{1} \lambda_{2}}}{n-\varepsilon_{\mathbf{k}}^{\lambda_{1}}}, \\
\left\langle\mathbf{k}, \lambda_{1}\left|\hat{\Gamma}_{\alpha}\right| \mathbf{k}, \lambda_{2}\right\rangle & =\Gamma_{\alpha}^{\lambda_{1} \lambda_{2}}(\mathbf{k}),
\end{aligned}
$$

where $\varepsilon_{\mathbf{k}}^{\lambda= \pm}=\zeta \alpha k_{x}+\lambda \varepsilon_{k}$, with $\varepsilon_{k}=\sqrt{k^{2}+m_{f}^{2}}$ the energy eigenvalue for the conduction $(\lambda=+)$ and valence $(\lambda=-)$ bands. We first perform the Matsubara summation over $n$ and subsequently an analytical continuation as $m_{j} \rightarrow \omega_{j}+i \delta$ with $\delta \rightarrow 0^{+}$. Eventually, we obtain

$$
\chi_{\alpha \beta \gamma}^{(2)}\left(\omega_{1}, \omega_{2}\right)=\sum_{\mathcal{P}}^{\prime} \sum_{\left\{\lambda_{i}\right\}} \sum_{\mathbf{k}} \frac{\Gamma_{\alpha}^{\lambda_{1} \lambda_{2}}(\mathbf{k}) \Gamma_{\beta}^{\lambda_{2} \lambda_{3}}(\mathbf{k}) \Gamma_{\gamma}^{\lambda_{3} \lambda_{1}}(\mathbf{k})}{\omega_{1}+\omega_{2}+\varepsilon_{\mathbf{k}}^{\lambda_{2}}-\varepsilon_{\mathbf{k}}^{\lambda_{1}}+i \delta}\left\{\frac{n_{\mathrm{F}}\left(\varepsilon_{\mathbf{k}}^{\lambda_{2}}\right)-n_{\mathrm{F}}\left(\varepsilon_{\mathbf{k}}^{\lambda_{3}}\right)}{\omega_{1}+\varepsilon_{\mathbf{k}}^{\lambda_{2}}-\varepsilon_{\mathbf{k}}^{\lambda_{3}}+i \delta}-\frac{n_{\mathrm{F}}\left(\varepsilon_{\mathbf{k}}^{\lambda_{3}}\right)-n_{\mathrm{F}}\left(\varepsilon_{\mathbf{k}}^{\lambda_{1}}\right)}{\omega_{2}+\varepsilon_{\mathbf{k}}^{\lambda_{3}}-\varepsilon_{\mathbf{k}}^{\lambda_{1}}+i \delta}\right\}
$$

where $n_{\mathrm{F}}(x)=1 /\left(e^{\beta(x-\mu)}+1\right)$ stands for the Fermi-Dirac distribution function. From now on, we use the shorthand notation $\omega_{j}+i \delta \rightarrow \omega_{j}$. We calculate $\chi_{y x x}(\omega, \omega)$ as the AC nonlinear Hall response to an external driving field along the $\hat{\mathbf{x}}$ direction. After summing over the band indices we find

$$
\chi_{y x x}^{(2)}(\omega, \omega)=-\sum_{\mathbf{k}, \zeta}\left\{n_{\mathrm{F}}\left(\varepsilon_{\mathbf{k}}^{c}\right)-n_{\mathrm{F}}\left(\varepsilon_{\mathbf{k}}^{v}\right)\right\}\left\{\frac{\left(\Gamma_{x}^{c c}-\Gamma_{x}^{v v}\right) \Gamma_{y}^{c v} \Gamma_{x}^{v c}}{\left(\varepsilon_{c v}-2 \omega\right)\left(\varepsilon_{c v}-\omega\right)}+\frac{\left(\Gamma_{x}^{c c}-\Gamma_{x}^{v v}\right) \Gamma_{x}^{c v} \Gamma_{y}^{v c}}{\left(\varepsilon_{c v}+2 \omega\right)\left(\varepsilon_{c v}+\omega\right)}+\frac{\left(\Gamma_{y}^{c c}-\Gamma_{y}^{v v}\right)\left|\Gamma_{x}^{c v}\right|^{2}}{\left(\varepsilon_{c v}-\omega\right)\left(\varepsilon_{c v}+\omega\right)}\right\}
$$

where $\varepsilon_{c v}=2 \varepsilon_{k}=2 \sqrt{m^{2}+k^{2}}$. We consider an electron-doped case where the integral over the entire valence band is zero because the integrand is an odd function of $\mathbf{k}$. For an arbitrary function $f(x)$ we have $\int_{0}^{2 \pi} d \phi \sin (\phi) f(\cos \phi)=0$. Using these two facts, we can make a further simplification

$$
\chi_{y x x}^{(2)}(\omega, \omega)=-i \omega \sum_{\zeta, \mathbf{k}} n_{\mathrm{F}}\left(\varepsilon_{\mathbf{k}}^{c}\right) \frac{\left(\Gamma_{x}^{c c}-\Gamma_{x}^{v v}\right) \operatorname{Im}\left[\Gamma_{y}^{c v} \Gamma_{x}^{v c}\right]}{\varepsilon_{c v}^{3}} \frac{6 \varepsilon_{c v}^{4}}{\varepsilon_{c v}^{4}-5 \varepsilon_{c v}^{2} \omega^{2}+4 \omega^{4}} .
$$

We recall that the Berry curvature reads

$$
\Omega_{y x}(\mathbf{k})=-\frac{2}{e^{2}} \frac{\operatorname{Im}\left[\Gamma_{y}^{c v} \Gamma_{x}^{v c}\right]}{\varepsilon_{c v}^{2}}=\frac{\zeta m_{f}}{2\left(m_{f}^{2}+k^{2}\right)^{3 / 2}} .
$$

Using the fact that $\Gamma_{x}^{c c}-\Gamma_{x}^{v v}=-e 2 \partial_{k_{x}} \varepsilon_{c v}$, we show that

$$
\frac{\left(\Gamma_{x}^{c c}-\Gamma_{x}^{v v}\right) \operatorname{Im}\left[\Gamma_{y}^{c v} \Gamma_{x}^{v c}\right]}{\varepsilon_{c v}^{3}}=\frac{e^{3}}{6} \partial_{k_{x}} \Omega_{y x}(\mathbf{k}) .
$$


Eventually, the conductivity can be formally written as [see Eq. (6)]

$$
\sigma_{y x x}^{(2)}(2 \omega)=-\frac{\chi_{y x x}^{(2)}(\omega, \omega)}{\omega^{2}}=\frac{i e^{3}}{\omega} \sum_{\mathbf{k}, \zeta} n_{\mathrm{F}}\left(\varepsilon_{\mathbf{k}}^{c}\right) \frac{\partial \Omega_{y x}(k)}{\partial k_{x}} C\left(\frac{\omega}{2 \varepsilon_{k}}\right),
$$

in which

$$
C(x)=\frac{1}{\left(1-4 x^{2}\right)\left(1-x^{2}\right)} .
$$

In the intraband regime $2 \omega \ll m_{f}$ we have $x \rightarrow 0$, which leads to $C(x) \rightarrow 1$. Therefore, the interband contribution is related to the factor equal to $C(x)-1$.

\section{APPENDIX C: ANALYTICAL FORM OF THE NONLINEAR HALL CONDUCTIVITY}

In this Appendix we perform the momentum integration in Eq. (B11). The contribution from two valleys are equal, which implies $\sum_{\zeta} \rightarrow 2$, and we set $\zeta=1$. At zero temperature we have $n_{\mathrm{F}}(x)=\Theta(\mu-x)$, where $\Theta(x)$ is the Heaviside step function. Therefore, we have the following relation for the nonlinear Hall conductivity:

$$
\sigma_{y x x}^{(2)}(2 \omega)=2 \frac{i e^{3}}{\omega} \sum_{\mathbf{k}} \Theta\left(\mu-\alpha k \cos (\phi)-\varepsilon_{k}\right) \frac{\partial \Omega_{y x}(\mathbf{k})}{\partial k_{x}} C\left(\frac{\omega}{2 \varepsilon_{k}}\right) .
$$

Considering the derivative of the Berry curvature as $\partial \Omega_{y x}(\mathbf{k}) / \partial k_{x}=-3 m_{f} k \cos \phi / 2 \varepsilon_{k}^{5}$, we find

$$
\sigma_{y x x}^{(2)}(2 \omega)=-\frac{3}{(2 \pi)^{2}} \frac{i e^{3}}{\omega} \int_{0}^{\infty} k d k\left[\int_{0}^{2 \pi} d \phi \cos (\phi) \Theta\left(\mu-\alpha k \cos (\phi)-\varepsilon_{k}\right)\right] \frac{m_{f} k}{\varepsilon_{k}^{5}} C\left(\frac{\omega}{2 \varepsilon_{k}}\right) .
$$

We then use the identity that holds for any real $a$ and $b$,

$$
\int_{0}^{2 \pi} d \phi \cos (\phi) \Theta(a-b \cos (\phi))=-2 \operatorname{sgn}(b) \Theta\left(1+\frac{a}{b}\right) \Theta\left(1-\frac{a}{b}\right) \sqrt{1-\left(\frac{a}{b}\right)^{2}}
$$

to obtain

$$
\sigma_{y x x}^{(2)}(2 \omega)=\frac{6}{(2 \pi)^{2}} \frac{i e^{3}}{\omega} \int_{0}^{\infty} k d k \Theta\left(\alpha k-\left(\mu-\varepsilon_{k}\right)\right) \Theta\left(\alpha k+\mu-\varepsilon_{k}\right) \sqrt{1-\left(\frac{\mu-\varepsilon_{k}}{\alpha k}\right)^{2}} \frac{m_{f} k}{\varepsilon_{k}^{5}} C\left(\frac{\omega}{2 \varepsilon_{k}}\right)
$$

Now we use another identity

$$
\begin{aligned}
\int_{0}^{\infty} d k \Theta\left(\alpha k-\left(\mu-\varepsilon_{k}\right)\right) \Theta\left(\alpha k+\mu-\varepsilon_{k}\right) g(\alpha, k) \\
\quad=\int_{k_{1}(\alpha)-k_{0}(\alpha)}^{k_{1}(\alpha)+k_{0}(\alpha)} d k g(\alpha, k)
\end{aligned}
$$

where

$$
k_{0}(\alpha)=\frac{\alpha \mu}{1-\alpha^{2}}, \quad k_{1}(\alpha)=\frac{\sqrt{\mu^{2}-m_{f}^{2}\left(1-\alpha^{2}\right)}}{1-\alpha^{2}} .
$$

Note that for $\mu>m_{f}>0$ and $0<\alpha<1$ we always have $k_{1}(\alpha)>k_{0}(\alpha)>0$. Therefore, we have

$$
\sigma_{y x x}^{(2)}(2 \omega)=\frac{i e^{3}}{\omega^{2}} f\left(\frac{\omega}{\mu}, \frac{m_{f}}{\mu}, \alpha\right),
$$

where the universal function $f(\cdots)$ is given by

$$
f\left(\frac{\omega}{\mu}, \frac{m_{f}}{\mu}, \alpha\right)=\frac{3 \omega m_{f}}{2 \pi^{2}} \int_{k_{1}(\alpha)-k_{0}(\alpha)}^{k_{1}(\alpha)+k_{0}(\alpha)} d k g(\alpha, k),
$$

where we define

$$
g(\alpha, k)=\frac{k / \alpha}{\varepsilon_{k}^{5}} \sqrt{(\alpha k)^{2}-\left(\mu-\varepsilon_{k}\right)^{2}} C\left(\frac{\omega}{2 \varepsilon_{k}}\right) .
$$

\section{Solution of the $f$ function for $\alpha \ll 1$}

For a very small $1 \gg \alpha>0$ we have

$$
\begin{aligned}
f & =\frac{3 \omega m_{f}}{2 \pi^{2}} \int_{k_{1}(\alpha)-k_{0}(\alpha)}^{k_{1}(\alpha)+k_{0}(\alpha)} d k g(\alpha, k) \\
& \approx \frac{3 \omega m_{f}}{2 \pi^{2}}\left\{2 k_{0}(\alpha) g\left(\alpha, k_{1}(\alpha)\right)\right\} .
\end{aligned}
$$

In this case, we can also approximate

$$
k_{0}(\alpha) \approx \alpha \mu, \quad k_{1}(\alpha) \approx \sqrt{\mu^{2}-m_{f}^{2}} .
$$

Accordingly, we arrive at the result given in Eq. (8):

$$
f \approx \frac{12 \alpha}{\pi^{2}} \frac{\omega m_{f}\left(\mu^{2}-m_{f}^{2}\right)}{\left(\mu^{2}-\omega^{2}\right)\left(4 \mu^{2}-\omega^{2}\right)} .
$$

\section{Solution of the $f$ function for an arbitrary value of $\alpha \leqslant 1$}

In this section we provide an exact solution for the $f$ function for arbitrary value of $\alpha \leqslant 1$. We recall that

$$
f=\frac{3 \omega m_{f}}{2 \pi^{2}} \int_{k_{1}(\alpha)-k_{0}(\alpha)}^{k_{1}(\alpha)+k_{0}(\alpha)} d k g(\alpha, k) .
$$



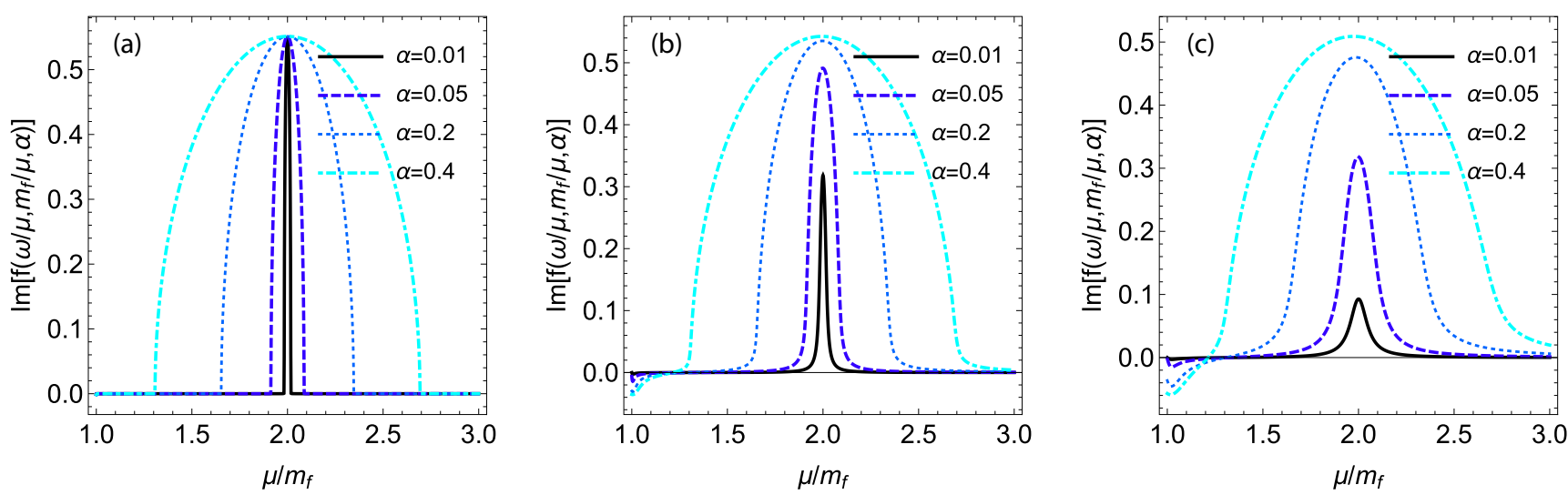

FIG. 5. Imaginary part of the universal $f$ function versus the chemical potential. We set $\omega=2 m_{f}$ and a resonance is clearly visible at $\mu=\omega$. An imaginary part for the frequency is introduced as $\omega \rightarrow \omega+i \Gamma$. We set (a) $\Gamma \rightarrow 0^{+}$, (b) $\Gamma=0.01 m_{f}$, and (c) $\Gamma=0.05 m_{f}$.

We introduce a new variable $y$ :

$$
y=\sqrt{m_{f}^{2}+k^{2}}, \quad y d y=k d k .
$$

Therefore, we have

$$
f=\frac{3 \omega m_{f}}{2 \pi^{2}} \frac{16}{\alpha} \int_{y_{-}(\alpha)}^{y_{+}(\alpha)} d y \frac{\sqrt{\alpha^{2}\left(y^{2}-m_{f}^{2}\right)-(\mu-y)^{2}}}{\left(y^{2}-\omega^{2}\right)\left(4 y^{2}-\omega^{2}\right)},
$$

where $y_{ \pm}(\alpha)=\sqrt{m_{f}^{2}+\left[k_{1}(\alpha) \pm k_{0}(\alpha)\right]^{2}}$. It can be shown that $\alpha^{2}\left(y^{2}-m_{f}^{2}\right)-(\mu-y)^{2}=\left(1-\alpha^{2}\right)\left\{a^{2}-\left(y-y_{0}\right)^{2}\right\}$, in which we define

$a=\alpha k_{1}(\alpha)=\frac{\alpha \sqrt{\mu^{2}-\left(1-\alpha^{2}\right) m_{f}^{2}}}{\left(1-\alpha^{2}\right)}, \quad y_{0}=\frac{k_{0}(\alpha)}{\alpha}=\frac{\mu}{1-\alpha^{2}}$.

After straightforward manipulation we find

$$
\begin{aligned}
f= & \frac{3 \omega m_{f}}{2 \pi^{2}} \frac{16 \sqrt{1-\alpha^{2}}}{6 \alpha \omega^{3}} \int_{y_{-}-y_{0}}^{y_{+}-y_{0}} d y \sqrt{a^{2}-y^{2}}\left\{\frac{1}{y+y_{0}-\omega}\right. \\
& \left.-\frac{1}{y+y_{0}+\omega}+\frac{2}{y+y_{0}+\omega / 2}-\frac{2}{y+y_{0}-\omega / 2}\right\} .
\end{aligned}
$$

The explicit form of the above function is obtained after solving the master integral

$$
F(y, a, b)=\int d y \frac{\sqrt{a^{2}-y^{2}}}{y+b}=F_{0}(y, a, b)+F_{1}(y, a, b),
$$

where

$$
\begin{aligned}
F_{0}(y, a, b)= & \sqrt{a^{2}-y^{2}}+b \arctan \left(\frac{y}{\sqrt{a^{2}-y^{2}}}\right), \\
F_{1}(y, a, b)= & \sqrt{a^{2}-b^{2}}[\ln (b+y) \\
& \left.-\ln \left(a^{2}+b y+\sqrt{a^{2}-b^{2}} \sqrt{a^{2}-y^{2}}\right)\right] .
\end{aligned}
$$

Eventually, we obtain

$$
f=\frac{4 \sqrt{1-\alpha^{2}}}{\pi^{2} \alpha} \frac{m_{f}\left[Q\left(y_{+}-y_{0}\right)-Q\left(y_{-}-y_{0}\right)\right]}{\omega^{2}},
$$

where

$$
\begin{aligned}
Q(y)= & F_{1}\left(y, a, y_{0}-\omega\right)-F_{1}\left(y, a, y_{0}+\omega\right) \\
& +2 F_{1}\left(y, a, y_{0}+\omega / 2\right)-2 F_{1}\left(y, a, y_{0}-\omega / 2\right) .
\end{aligned}
$$

It is worth noting that the contribution from $F_{0}(y, a, b)$ in the end cancels out in the $Q$ function. Equations (C21) and (C7) together yield the nonlinear conductivity in the bare bubble level. The numerical plots given in Fig. 2 are generated by using Eq. (C21).

\section{APPENDIX D: NON-LORENTZIAN VERSUS LORENTZIAN RESONANCE}

The nonlinear Hall conductivity is given in Eq. (7) in which the universal function $f\left(\omega / \mu, m_{f} / \mu, \alpha\right)$ shows interesting interband features. The interband resonance peak becomes broader when the value of the tilt parameter is increased while there is no scattering mechanism in the model (we consider the collisionless regime). Intriguingly, we can see the difference of this resonance with the usual Lorentzian one after including a finite imaginary part for the frequency, e.g., $\omega \rightarrow \omega+i \Gamma$. The shape of the resonances for different values of $\alpha$ and $\Gamma$ are illustrated in Figs. 5 and 6. For small $\alpha$ and finite $\Gamma$ the resonances feature a Lorentzian shape, while by increasing the value of $\alpha$ they become broader without a considerable change in the peak value. The Lorentzian function changes its curvature sign from negative to positive when we move away from the resonance [see Fig. 5(c)], while in the non-Lorentzian case the curvature is always negative [see Fig. 5(a)].

\section{APPENDIX E: DETAILS OF THE RENORMALIZATION-GROUP ANALYSIS OF THE GROSS-NEVEU-YUKAWA THEORY FOR THE TILTED DIRAC FERMIONS}

For the sake of completeness, we repeat here some of the details already presented at the beginning of Sec. IV. The momentum-imaginary-time action for the noninteracting tilted Dirac fermions in $D$ spatial dimensions reads

$$
S_{f}=\int d \tau d^{D} \mathbf{r} \psi^{\dagger}(\tau, \mathbf{r})\left[\partial_{\tau}+\hat{\mathcal{H}}\left(\mathbf{k} \rightarrow-i \nabla_{\mathbf{r}}\right)\right] \psi(\tau, \mathbf{r}) .
$$



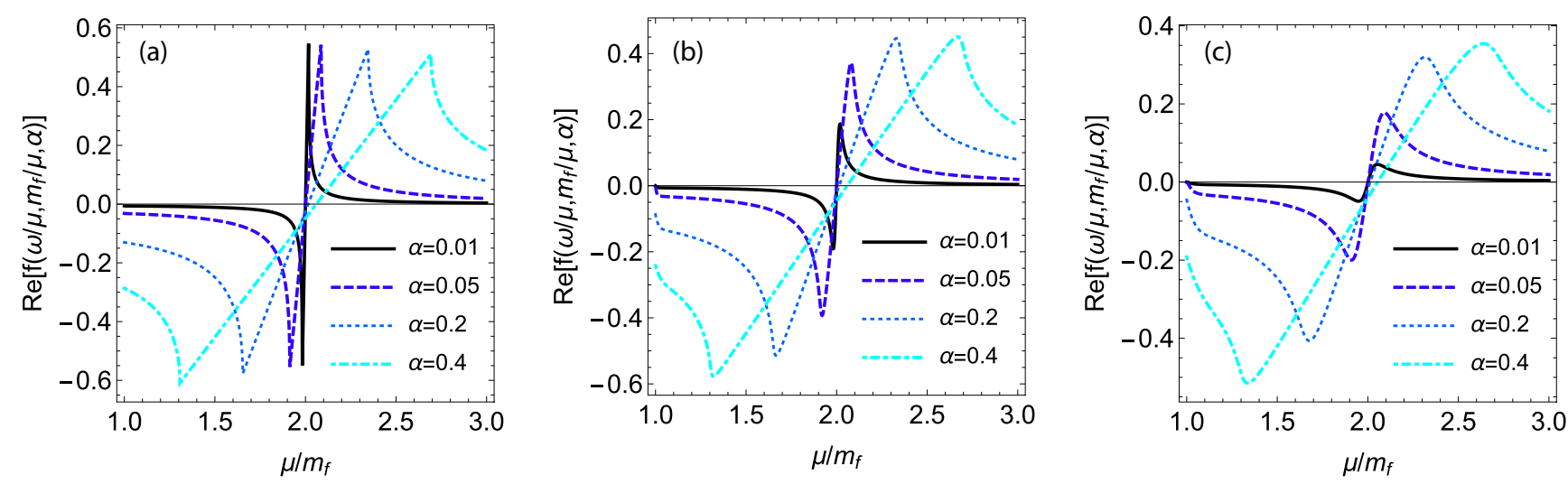

FIG. 6. Real part of the universal $f$ function versus the chemical potential. We set $\omega=2 m_{f}$ and a resonance is clearly visible at $\mu=\omega$. An imaginary part for the frequency is introduced as $\omega \rightarrow \omega+i \Gamma$. We set (a) $\Gamma \rightarrow 0^{+}$, (b) $\Gamma=0.01 m_{f}$, and (c) $\Gamma=0.05 m_{f}$.

Here $\hat{\mathcal{H}}(\mathbf{k})$ is the real-space Hamiltonian for the free tilted massive Dirac fermions obtained from its momentum space representation given by

$$
\hat{\mathcal{H}}(\mathbf{k})=\alpha \zeta k_{x} \hat{I}+v\left(\zeta k_{x} \hat{\sigma}_{x}+k_{y} \hat{\sigma}_{y}\right)+m_{f} \hat{\sigma}_{z},
$$

where $\zeta= \pm$ stands for two fermion flavors, $m_{f}$ represents the fermion mass due to the inversion-symmetry breaking, $\hat{\sigma}$ are the Pauli matrices, $\hat{I}$ is the $2 \times 2$ unity matrix, $|\alpha|<1$ is the tilt parameter, and the Fermi velocity $v=1$ hereafter. The short-range interaction is encoded through the Yukawa coupling between the Dirac fermion quasiparticles and the bosonic degrees of freedom representing the fluctuations of the underlying ordered state, assumed to be a charge density wave for simplicity, and reads

$$
S_{Y}=g \int d \tau \int d^{D} \mathbf{r} \phi(\tau, \mathbf{r}) \psi^{\dagger}(\tau, \mathbf{r}) \hat{\sigma}_{z} \psi(\tau, \mathbf{r}) .
$$

The dynamics of the bosonic fluctuations is described by the action

$$
S_{b}=\int d \tau \int d^{D} \mathbf{r}\left\{\phi(\tau, \mathbf{r})\left(-\partial_{\tau}^{2}-\nabla^{2}+m_{b}^{2}\right) \phi(\tau, \mathbf{r})+\frac{\lambda}{4 !} \phi^{4}\right\},
$$

where $m_{b}^{2}$ is the bosonic mass scaling as the distance from the QCP and $m_{b}^{2}$ is the tuning parameter for the QPT. It can be readily shown that for each of the valleys $\zeta= \pm$, the above action can be cast in the manifestly Lorentz-invariant form, except for the tilt term, which explicitly breaks this symmetry. For instance, taking $\zeta=+$, to obtain such a form of the action, we can choose the $2 \times 2$ Dirac $\gamma$ matrices as $\gamma_{0}=\hat{\sigma}_{z}, \gamma_{1}=-\hat{\sigma}_{y}, \gamma_{2}=\hat{\sigma}_{x}$, and $\bar{\psi}=\psi^{\dagger} \gamma_{0}$. In particular, the Yukawa term in Eq. (E3) can be rewritten in the relativistic form as $S_{Y}=g \int d \tau \int d^{D} \mathbf{r} \phi \bar{\psi} \psi$. Taking into account the other valley, the basis of the $4 \times 4 \gamma$ matrices can be chosen as $\Gamma_{\mu}=\left\{\Gamma_{0}, \Gamma_{1}, \Gamma_{2}\right\}=\left\{\hat{\sigma}_{z} \otimes \hat{\tau}_{0},-\hat{\sigma}_{y} \otimes \hat{\tau}_{z}, \hat{\sigma}_{x} \otimes \hat{\tau}_{0}\right\}$, with the $\hat{\tau}$ matrices acting in the valley space and $\hat{\tau}_{0}$ as the $2 \times 2$ unity matrix. In this basis the fermionic part of the action including now both (decoupled) valleys takes the Lorentz invariant form (except for the tilt term).

Engineering scaling dimensions in the units of momentum (inverse length) of the Yukawa and $\phi^{4}$ couplings are $\operatorname{dim}\left[g^{2}\right]=\operatorname{dim}[\lambda]=3-D$, while $\operatorname{dim}[\alpha]=0$, and therefore $D=3$ is the upper critical dimension in the theory. We will therefore use $\epsilon=3-D$, the deviation from the upper critical dimension as an expansion parameter to access quantumcritical behavior in $D=2$.

\section{Fermionic self-energy}

To find the correction to the nonlinear conductivity close to the QCP we compute the leading-order zero-temperature $(T=0)$ self-energy for the tilted Dirac fermions [see Fig. 7(a)]

$$
\begin{aligned}
\Sigma_{f, \zeta}(i \Omega, \mathbf{k})= & g^{2} \int[d \omega] \int\left[d^{D} \mathbf{q}\right] \hat{\sigma}_{z} G_{f, \zeta}(i \omega+i \Omega, \mathbf{k}+\mathbf{q}) \\
& \times \hat{\sigma}_{z} G_{b}(i \omega, \mathbf{q}),
\end{aligned}
$$

where $[d \omega] \equiv d \omega / 2 \pi, \quad\left[d^{D} \mathbf{k}\right] \equiv d^{D} \mathbf{k} /(2 \pi)^{D}, \quad$ and the fermionic and bosonic propagators read, respectively,

$$
\begin{gathered}
G_{f}(i \omega, \mathbf{k})=\frac{i \omega+\alpha \zeta k_{x}+\tau \hat{\sigma}_{x} k_{x}+\hat{\sigma}_{y} k_{y}+m_{f} \hat{\sigma}_{z}}{\left(\omega+i \alpha \tau k_{x}\right)^{2}+k^{2}+m_{f}^{2}}, \\
G_{b}(i \omega, \mathbf{k})=\frac{1}{\omega^{2}+k^{2}+m_{b}^{2}}
\end{gathered}
$$

and we set the bosonic and fermionic velocities to be equal to unity in the critical region [37]. After performing the matrix algebra in $D=2$, the above self-energy in the critical hyperplane (all masses set to zero) explicitly reads

$$
\Sigma_{f, \zeta}(i \Omega, \mathbf{k})=g^{2} \int[d \omega]\left[d^{D} \mathbf{q}\right] \frac{i(\omega+\Omega)-\alpha \zeta\left(k_{x}+q_{x}\right)-\zeta \hat{\sigma}_{x}\left(k_{x}+q_{x}\right)-\hat{\sigma}_{y}\left(k_{y}+q_{y}\right)}{\left\{\left[\omega+\Omega+i \alpha \zeta\left(k_{x}+q_{x}\right)\right]^{2}+(\mathbf{k}+\mathbf{q})^{2}\right\}\left(\omega^{2}+q^{2}\right)} .
$$

We now set $\mathbf{k}=0$ and $\zeta=1$ to obtain

$$
\Sigma_{f}(i \Omega, 0)=-g^{2} \int \frac{d^{D} \mathbf{q}}{(2 \pi)^{D}} \int_{-\infty}^{\infty} \frac{d \omega}{2 \pi} \frac{i(\omega+\Omega)-\boldsymbol{\alpha} \cdot \mathbf{q}-\boldsymbol{\sigma} \cdot \mathbf{q}}{\left\{[i(\omega+\Omega)-\boldsymbol{\alpha} \cdot \mathbf{q}]^{2}-q^{2}\right\}\left(\omega^{2}+q^{2}\right)},
$$




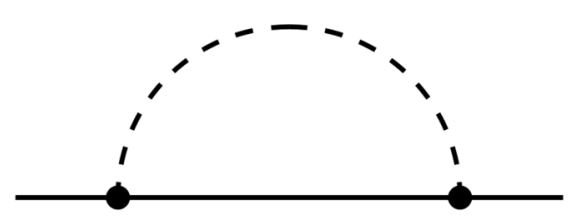

(a)

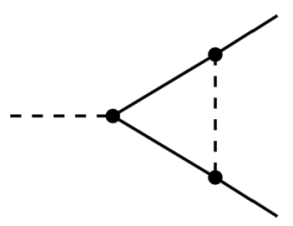

(c)

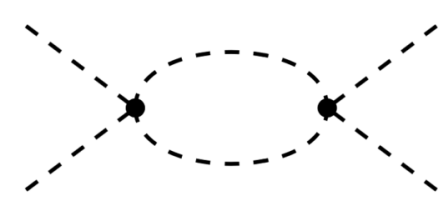

(d)

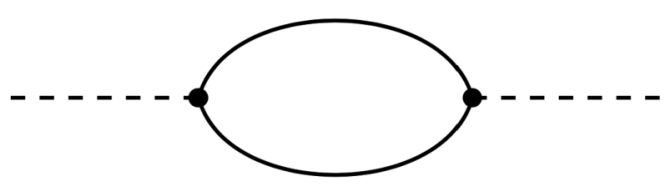

(b)

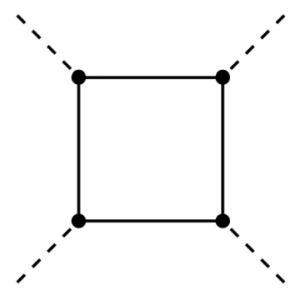

(e)

FIG. 7. Feynman diagrams to the leading order in the $\epsilon$ expansion: (a) fermionic self-energy, (b) bosonic self-energy, (c) correction to the Yukawa vertex, (d) correction to the $\lambda$ vertex from the bosonic quartic coupling, and (e) correction to the $\lambda$ vertex from the Yukawa interaction. The solid line represents the fermionic propagator while the dashed line stands for the bosonic propagator.

with $\boldsymbol{\alpha} \equiv \alpha \hat{\mathbf{x}}$ and $\hat{\mathbf{x}}$ the unit vector in the $x$ direction. We perform the integral over $\omega$,

$$
\Sigma_{f}(i \Omega, 0)=-\frac{g^{2}}{2} \int \frac{d^{D} \mathbf{q}}{(2 \pi)^{D}} \frac{1}{q} \frac{i \Omega-\boldsymbol{\alpha} \cdot \mathbf{q}-\boldsymbol{\sigma} \cdot \mathbf{q}}{(i \Omega-\boldsymbol{\alpha} \cdot \mathbf{q})^{2}-4 q^{2}} .
$$

Next we drop the term proportional to $\boldsymbol{\sigma} \cdot \mathbf{q}$, since it can only generate a term of the form $\boldsymbol{\sigma} \cdot \boldsymbol{\alpha}$, which we eliminate by the corresponding counterterm, to find

$$
\Sigma_{f}(i \Omega, 0)=-\frac{g^{2}}{2} \int \frac{d^{D} \mathbf{q}}{(2 \pi)^{D}} \frac{1}{q} \frac{i \Omega-\boldsymbol{\alpha} \cdot \mathbf{q}}{(i \Omega-\boldsymbol{\alpha} \cdot \mathbf{q})^{2}-4 q^{2}},
$$

which yields

$$
\lim _{i \Omega \rightarrow 0} \frac{\partial \Sigma_{f}(i \Omega, 0)}{\partial(i \Omega)}=\frac{g^{2}}{2} \int \frac{d^{D} \mathbf{q}}{(2 \pi)^{D}} \frac{1}{q} \frac{4 q^{2}+(\boldsymbol{\alpha} \cdot \mathbf{q})^{2}}{\left[4 q^{2}-(\boldsymbol{\alpha} \cdot \mathbf{q})^{2}\right]^{2}} .
$$

We then integrate over the momentum using the hard cutoff $\Lambda$ in $D=3$ and use that $\alpha=\alpha \hat{\mathbf{x}}$ to find

$$
\lim _{i \Omega \rightarrow 0} \frac{\partial \Sigma_{f}(i \Omega, 0)}{\partial(i \Omega)}=\frac{g^{2}}{(4 \pi)^{2}} G(\alpha) \int_{\lambda}^{\Lambda} \frac{d q}{q},
$$

with $\lambda=\Lambda / b$. Here $b>1$ but $b-1 \ll 0$ is the Wilsonian RG parameter. Finally, we use the correspondence between the hard cutoff and the dimensional regularizations

$$
\int_{\lambda}^{\Lambda} \frac{d q}{q} \rightarrow \frac{1}{\epsilon}
$$

which can be explicitly checked, for instance, by setting $\alpha=0$ in Eq. (E9), and keep only the divergent piece in the self-energy. This procedure is systematically used in our analysis. Furthermore, we define

$$
\begin{aligned}
G(\alpha) & =2 \int_{0}^{2 \pi} \frac{d \phi}{2 \pi} \int_{0}^{\pi} d \theta \sin \theta \frac{4+\alpha^{2}(\sin \theta \cos \phi)^{2}}{\left[4-\alpha^{2}(\sin \theta \cos \phi)^{2}\right]^{2}} \\
& =4 \int_{0}^{\pi} d \theta \sin \theta\left(4-\alpha^{2} \sin ^{2} \theta\right)^{-3 / 2} \\
& =\frac{4}{4-\alpha^{2}}=\frac{1}{1-\alpha^{2} / 4} .
\end{aligned}
$$

Note that this function is strictly positive for $|\alpha|<1$.

We then obtain the fermion field renormalization constant

$$
Z_{\Psi}=1-\lim _{i \Omega \rightarrow 0} \frac{\partial \Sigma_{f}(i \Omega, 0)}{\partial(i \Omega)}=1-\frac{g^{2}}{(4 \pi)^{2}} \frac{1}{\epsilon} G(\alpha) .
$$

We now calculate $Z_{\alpha}$ using the condition

$$
-Z_{\Psi} Z_{\alpha} \alpha k_{x}-\left.k_{x} \frac{\partial \Sigma_{f}(i \Omega, \mathbf{k})}{\partial k_{x}}\right|_{i \Omega \rightarrow 0, \mathbf{k} \rightarrow 0}=-\alpha k_{x} .
$$

We use Eq. (E10) for a finite external momentum $\mathbf{k}$, obtained by shifting $\mathbf{q} \rightarrow \mathbf{q}+\mathbf{k}$, which then yields

$$
\left.\frac{\partial \Sigma_{f}(i \Omega, \mathbf{k})}{\partial k_{x}}\right|_{i \Omega \rightarrow 0, \mathbf{k} \rightarrow 0}=\frac{g^{2}}{(4 \pi)^{2}} \alpha G(\alpha) \frac{1}{\epsilon},
$$

with $G(\alpha)$ given by Eq. (E15). Therefore, using this result, the already obtained $Z_{\Psi}$ in Eq. (E16), and Eq. (E17), we find that $Z_{\alpha}=1$, implying that the parameter $\alpha$ remains marginal to the leading order in the $\epsilon$ expansion.

\section{Self-energy for the bosonic field}

To find the RG flow equation for the Yukawa coupling, we compute the self-energy for the bosonic field and the correction to the Yukawa vertex. We first compute the 
self-energy for the bosons [Fig. 7(b)], which reads

$$
\Sigma_{b}(i \Omega, \mathbf{k})=-2 g^{2} \int[d \omega] \int\left[d^{D} \mathbf{q}\right] \operatorname{tr}\left[\sigma_{z} G_{f}(i \omega, \mathbf{q}) \sigma_{z} G_{f}(i \omega+i \Omega, \mathbf{q}+\mathbf{k})\right] .
$$

Since we are after the wave-function renormalization of the bosonic field, we set $\mathbf{k}=0$ in the above self-energy. After taking the trace, introducing the Feynman parameter, and shifting the integral over the frequency, we obtain

$$
\Sigma_{b}(i \Omega, \mathbf{k}=0)=4 g^{2} N_{f} \int_{0}^{1} d x \int[d \omega] \int\left[d^{D} \mathbf{q}\right] \frac{(\omega-x \Omega)[\omega+(1-x) \Omega]+q^{2}}{\left[\omega^{2}+(1-x) 2 i \alpha \Omega q_{x}+x(1-x) \Omega^{2}+q^{2}\right]^{2}},
$$

with $2 N_{f}$ as the number of two-component Dirac spinors. The bosonic wave-function renormalization is determined by the renormalization condition

$$
Z_{\Phi}-\left.\frac{\partial \Sigma_{b}(i \Omega, 0)}{\partial\left(\Omega^{2}\right)}\right|_{\Omega \rightarrow 0}=1 .
$$

After taking the derivative and performing the remaining integrals, we obtain

$$
Z_{\Phi}=1-\frac{g^{2}}{8 \pi^{2}} 2 N_{f}\left(1+2 \alpha^{2}\right) \frac{1}{\epsilon} .
$$

\section{The $\beta$ function for Yukawa coupling}

We now compute the correction to the Yukawa vertex [Fig. 7(c); we take $\zeta=1$ for the valley index]

$$
\begin{aligned}
\delta g= & -g^{3} \int[d \omega] \int[d \mathbf{q}] \hat{\sigma}_{z} G_{f}(i \omega, \mathbf{q}) \hat{\sigma}_{z} G_{f}(i \omega+i \Omega, \mathbf{q}+\mathbf{k}) \\
& \times \hat{\sigma}_{z} G_{b}(i \omega, \mathbf{q}) .
\end{aligned}
$$

After setting the external momentum and frequency to zero, since we are only after the divergent piece of the integral, we obtain

$$
\delta g=g^{3} \hat{\sigma}_{z} \int[d \omega] \int[d \mathbf{q}] \frac{1}{\left[\left(\omega+i \alpha q_{x}\right)^{2}+q^{2}\right]\left(\omega^{2}+q^{2}\right)},
$$

which, after carrying out the frequency and the momentum integrals, in turn yields

$$
\delta g=g^{3} \hat{\sigma}_{z} \frac{1}{8 \pi^{2}} V(\alpha) \frac{1}{\epsilon},
$$

where

$$
V(\alpha)=\frac{2}{|\alpha|} \operatorname{arccosh}\left(\frac{2}{\sqrt{4-\alpha^{2}}}\right) .
$$

The renormalization condition for the Yukawa coupling reads

$$
Z_{\Psi} Z_{\Phi}^{1 / 2} g \hat{\sigma}_{z}-\delta g=g_{0} \kappa^{-\epsilon / 2} \hat{\sigma}_{z},
$$

where $\kappa$ is the RG momentum scale [20]. Using that the bare coupling $g_{0}$ is stationary under the RG transformation, we find the infrared $\beta$ function for the Yukawa coupling $\beta_{g^{2}} \equiv-\frac{d g^{2}}{d \ln \kappa}$,

$$
\begin{aligned}
\beta_{g^{2}} & =\epsilon g^{2}-g^{4}\left[G(\alpha)+2 V(\alpha)+2 N_{f}\left(1+2 \alpha^{2}\right)\right] \\
& \equiv \epsilon g^{2}-g^{4} K\left(\alpha, N_{f}\right),
\end{aligned}
$$

with

$$
K\left(\alpha, N_{f}\right)=G(\alpha)+2 V(\alpha)+2 N_{f}\left(1+2 \alpha^{2}\right) .
$$

Here we use that $\alpha$ is a marginal parameter to the leading order in the $\epsilon$ expansion and rescale $g^{2} / 8 \pi^{2} \rightarrow g^{2}$. In the limit $\alpha \rightarrow 0$ we obtain the known result for the $\beta$ function for the Yukawa coupling in the Ising GNY theory $[22,38]$

$$
\beta_{g^{2}}=\epsilon g^{2}-2 g^{4}\left(N_{f}+\frac{3}{2}\right) .
$$

For $|\alpha|<1$ different from zero, the location of the critical point

$$
g_{\star}^{2}=\frac{\epsilon}{K\left(\alpha, N_{f}\right)}
$$

is always pushed down in comparison to the case without the tilting $(\alpha=0)$, since the function $K\left(\alpha, N_{f}\right)$ defined in Eq. (E28) is positive, even, and monotonically increasing for $0<\alpha<1$.

\section{The $\beta$ function for $\lambda$ coupling}

The vertex correction of the $\lambda$ coupling for tilted Dirac fermions turns out to be equal to the case $\alpha=0$. Namely, the renormalization of the $\phi^{4}$ vertex does not depend on $\alpha$ as the bosonic propagator is $\alpha$ independent [see Fig. 7(d)] and reads

$$
\delta \lambda_{\lambda}=\frac{3}{2} \frac{\lambda^{2}}{8 \pi^{2}} \frac{1}{\epsilon} .
$$

Furthermore, the explicit calculation of the correction of the $\lambda$ coupling due to the Yukawa interaction [Fig. 7(e)] gives the same result as without the tilt. This is a consequence of the fact that the tilt parameter can be eliminated in the corresponding integral by the shift of the frequency and the
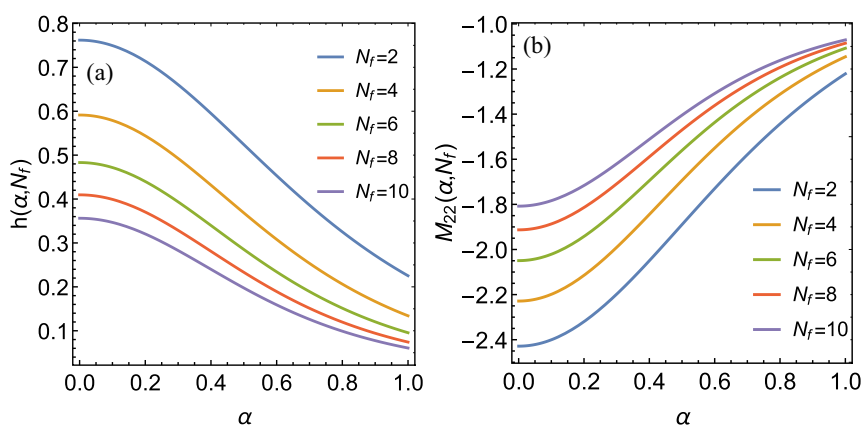

FIG. 8. (a) Function $h\left(\alpha, N_{f}\right)$ determining the value of the coupling $\lambda$ at the fixed point $X_{\star}=\left\{g_{\star}^{2}, \lambda_{\star}\right\}, \lambda_{\star}=\epsilon h\left(\alpha, N_{f}\right)$, of the RG flow given by Eqs. (E28) and (E35), with $g_{\star}^{2}$ given in the text. (b) Eigenvalue $M_{22}$ of the stability matrix $M_{i j}(X)$ at the fixed point $X=X_{\star}$, showing that indeed the $\lambda$ direction is stable and therefore $X_{\star}$ is a QCP at least for the values of $N_{f} \leqslant 10$. 
fact that the diagram is calculated in the limit of the vanishing external momentum and frequency. Explicitly, the divergent part of this contribution reads

$$
\begin{aligned}
\delta \lambda_{Y}= & -\frac{24 g^{4}}{4} \int[d \omega] \int\left[d^{D} \mathbf{q}\right] \operatorname{tr}\left[\hat{\sigma}_{z} G_{f}(i \omega, \mathbf{q}) \hat{\sigma}_{z} G_{f}(i \omega, \mathbf{q})\right. \\
& \left.\times \hat{\sigma}_{z} G_{f}(i \omega, \mathbf{q}) \hat{\sigma}_{z} G_{f}(i \omega, \mathbf{q})\right] \\
= & -24 N_{f} \frac{g^{4}}{8 \pi^{2}} \frac{1}{\epsilon} .
\end{aligned}
$$

The renormalization condition of the $\lambda$ coupling reads

$$
Z_{\Phi}^{2} \lambda-\left(\delta \lambda_{\lambda}+\delta \lambda_{Y}\right)=\lambda_{0} \kappa^{-\epsilon}
$$

where $\lambda_{0}$ is the bare coupling and the term on the right-hand side accounts for the scaling dimension of the $\lambda$ coupling $\operatorname{dim}[\lambda]=3-D=\epsilon$. Using that the bare coupling is stationary under the RG transformation, and the results in Eqs. (E32) and (E33), we obtain the infrared $\beta$ function for the $\phi^{4}$ coupling

$$
\beta_{\lambda}=\epsilon \lambda-\frac{3}{2} \lambda^{2}-4 N_{f}\left(1+2 \alpha^{2}\right) \lambda g^{2}+24 g^{4} N_{f},
$$

which in the limit $\alpha=0$ agrees with the known result for the $Z_{2}$ GNY theory for the untilted Dirac fermions [22,38]. Substituting the value of the critical Yukawa coupling given by Eq. (E31) into the above $\beta$ function for the $\lambda$ coupling, we find that the fixed point is located at $\lambda_{\star}=\epsilon h\left(\alpha, N_{f}\right)$, with $h\left(\alpha, N_{f}\right)$ a complicated function of its arguments, plotted in Fig. 8(a) as a function of the tilt parameter for different values of $N_{f}$. The value of $\lambda_{\star}$ for $\alpha \neq 0$ is smaller than that for $\alpha=0$, as expected from the scaling of the density of states for the TDFs. The fixed point $X_{\star} \equiv\left(g_{\star}^{2}, \lambda_{\star}\right)$ is in fact a critical point since the eigenvalues of the stability matrix $M_{i j}(X)=\partial \beta_{X_{i}} / \partial X_{j}$, where $X \equiv\left\{g^{2}, \lambda\right\}$, equal to its diagonal elements, are both negative at $X=X_{\star}$. The eigenvalue $M_{11}\left(X_{\star}\right)=-\epsilon K\left(\alpha, N_{f}\right)<0$, since the function $K\left(\alpha, N_{f}\right)$ is positive for $|\alpha|<1$. The other eigenvalue $M_{22}\left(X_{\star}\right)$ is also negative, as can be seen in Fig. 8(b).
[1] Y. Shen, The Principles of Nonlinear Optics (WileyInterscience, New York, 2003).

[2] E. Deyo, L. E. Golub, E. L. Ivchenko, and B. Spivak, arXiv:0904.1917.

[3] J. E. Moore and J. Orenstein, Phys. Rev. Lett. 105, 026805 (2010).

[4] P. Hosur, Phys. Rev. B 83, 035309 (2011).

[5] I. Sodemann and L. Fu, Phys. Rev. Lett. 115, 216806 (2015).

[6] Q. Ma, S.-Y. Xu, H. Shen, D. MacNeill, V. Fatemi, T.-R. Chang, A. M. Mier Valdivia, S. Wu, Z. Du, C.-H. Hsu, S. Fang, Q. D. Gibson, K. Watanabe, T. Taniguchi, R. J. Cava, E. Kaxiras, H.-Z. Lu, H. Lin, L. Fu, N. Gedik, and P. Jarillo-Herrero, Nature (London) 565, 337 (2019).

[7] S.-Y. Xu, Q. Ma, H. Shen, V. Fatemi, S. Wu, T.-R. Chang, G. Chang, A. M. M. Valdivia, C.-K. Chan, Q. D. Gibson, J. Zhou, Z. Liu, K. Watanabe, T. Taniguchi, H. Lin, R. J. Cava, L. Fu, N. Gedik, and P. Jarillo-Herrero, Nat. Phys. 14, 900 (2018).

[8] K. Kang, T. Li, E. Sohn, J. Shan, and K. F. Mak, Nat. Mater. 18, 324 (2019).

[9] Y. Zhang, Y. Sun, and B. Yan, Phys. Rev. B 97, 041101(R) (2018).

[10] J.-S. You, S. Fang, S.-Y. Xu, E. Kaxiras, and T. Low, Phys. Rev. B 98, 121109(R) (2018).

[11] J. Quereda, T. S. Ghiasi, J.-S. You, J. van den Brink, B. J. van Wees, and C. H. van der Wal, Nat. Commun. 9, 3346 (2018).

[12] J. I. Facio, D. Efremov, K. Koepernik, J.-S. You, I. Sodemann, and J. van den Brink, Phys. Rev. Lett. 121, 246403 (2018).

[13] F. de Juan, A. G. Grushin, T. Morimoto, and J. E. Moore, Nat. Commun. 8, 15995 (2017).

[14] Q. Ma, S.-Y. Xu, C.-K. Chan, C.-L. Zhang, G. Chang, Y. Lin, W. Xie, T. Palacios, H. Lin, S. Jia, P. A. Lee, P. Jarillo-Herrero, and N. Gedik, Nat. Phys. 13, 842 (2017).

[15] H. Rostami and M. Polini, Phys. Rev. B 97, 195151 (2018).

[16] F. de Juan, Y. Zhang, T. Morimoto, Y. Sun, J. E. Moore, and A. G. Grushin, arXiv:1907.02537.
[17] O. Matsyshyn and I. Sodemann, Phys. Rev. Lett. 123, 246602 (2019).

[18] R. Shankar, Rev. Mod. Phys. 66, 129 (1994).

[19] B. Roy and V. Juričić, Phys. Rev. B 99, 121407(R) (2019).

[20] J. Zinn-Justin, Quantum Field Theory and Critical Phenomena (Oxford University Press, Oxford, 2002).

[21] B. Rosenstein and A. Kovner, Phys. Lett. B 314, 381 (1993).

[22] I. F. Herbut, V. Juričić, and O. Vafek, Phys. Rev. B 80, 075432 (2009).

[23] B. Roy, Phys. Rev. B 84, 113404 (2011).

[24] B. Roy, V. Juričić, and I. F. Herbut, Phys. Rev. B 87, 041401(R) (2013).

[25] P. Ponte and S.-S. Lee, New J. Phys. 16, 013044 (2014).

[26] B. Roy and V. Juričić, Phys. Rev. B 90, 041413(R) (2014).

[27] S.-K. Jian, Y.-F. Jiang, and H. Yao, Phys. Rev. Lett. 114, 237001 (2015).

[28] S. Sorella, Y. Otsuka, and S. Yunoki, Sci. Rep. 2, 992 (2012).

[29] F. F. Assaad and I. F. Herbut, Phys. Rev. X 3, 031010 (2013).

[30] F. Parisen Toldin, M. Hohenadler, F. F. Assaad, and I. F. Herbut, Phys. Rev. B 91, 165108 (2015).

[31] Y. Otsuka, S. Yunoki, and S. Sorella, Phys. Rev. X 6, 011029 (2016).

[32] S. Sachdev, Quantum Phase Transitions, 2nd ed. (Cambridge University Press, Cambridge, 2011).

[33] B. Roy and V. Juričić, Phys. Rev. Lett. 121, 137601 (2018).

[34] S. Katayama, A. Kobayashi, and Y. Suzumura, J. Phys. Soc. Jpn. 75, 054705 (2006).

[35] T. Nishine, A. Kobayashi, and Y. Suzumura, J. Phys. Soc. Jpn. 79, 114715 (2010).

[36] D. Xiao, M.-C. Chang, and Q. Niu, Rev. Mod. Phys. 82, 1959 (2010).

[37] B. Roy, V. Juričić, and I. F. Herbut, J. High Energy Phys. 04 (2016) 18

[38] B. Roy, P. Goswami, and V. Juričić, Phys. Rev. B 97, 205117 (2018).

[39] T. S. Sikkenk and L. Fritz, Phys. Rev. B 100, 085121 (2019). 
[40] L.-k. Shi and J. C. W. Song, Phys. Rev. B 99, 035403 (2019).

[41] Z. Z. Du, C. M. Wang, H.-Z. Lu, and X. C. Xie, Phys. Rev. Lett. 121, 266601 (2018).

[42] X.-F. Zhou, X. Dong, A. R. Oganov, Q. Zhu, Y. Tian, and H.-T. Wang, Phys. Rev. Lett. 112, 085502 (2014).

[43] A. J. Mannix, X.-F. Zhou, B. Kiraly, J. D. Wood, D. Alducin, B. D. Myers, X. Liu, B. L. Fisher, U. Santiago, J. R. Guest, M. J. Yacaman, A. Ponce, A. R. Oganov, M. C. Hersam, and N. P. Guisinger, Science 350, 1513 (2015).

[44] T. Farajollahpour, Z. Faraei, and S. A. Jafari, Phys. Rev. B 99, 235150 (2019).

[45] Y. Tanaka, Z. Ren, T. Sato, K. Nakayama, S. Souma, T. Takahashi, K. Segawa, and Y. Ando, Nat. Phys. 8, 800 (2012).
[46] K. Kajita, T. Ojiro, H. Fujii, Y. Nishio, H. Kobayashi, A. Kobayashi, and R. Kato, J. Phys. Soc. Jpn. 61, 23 (1992).

[47] N. Tajima, M. Tamura, Y. Nishio, K. Kajita, and Y. Iye, J. Phys. Soc. Jpn. 69, 543 (2000).

[48] M. Hirata, K. Ishikawa, G. Matsuno, A. Kobayashi, K. Miyagawa, M. Tamura, C. Berthier, and K. Kanoda, Science 358, 1403 (2017).

[49] B. Roy and V. Juričić, Phys. Rev. B 96, 155117 (2017).

[50] G. D. Mahan, Many-Particle Physics, 3rd ed., Physics of Solids and Liquids (Springer, New York, 2000).

[51] P. N. Butcher and D. Cotter, The Elements of Nonlinear Optics (Cambridge University Press, Cambridge, 1990). 